\title{
Pasting properties by near-infrared reflectance analysis of whole grain paddy rice samples
}

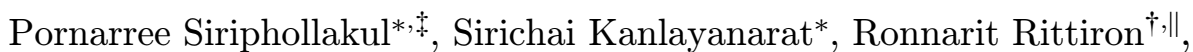 \\ Jaitip Wanitchang*, Thongchai Suwonsichon ${ }^{\S}$, Panida Boonyaritthongchai* \\ and Kazuhiro Nakano \\ *Division of Postharvest Technology \\ KingMongkut's University of Technology Thonburi 126 Pracha-Utid Road \\ Bangmod, Toongkru, Bangkok 10140, Thailand \\ ${ }^{\dagger}$ Department of Food Engineering, Faculty of Engineering at Kamphaengsaen \\ Kasetsart University, Nakhonpathom, Thailand \\ \$Division of Agricultural Engineering and Technology \\ Rajamungala University of Technology \\ Tawan-ok, 43/6 Bangpha, Sriracha, Chonburi, Thailand

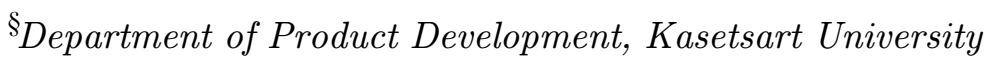 \\ 50 Ngam Wong Wan Rd Ladyao, Chatuchak Bangkok, Thailand \\ "Graduate School of Science and Technology, Niigata University \\ 8050 Ikarashi 2-no-cho Nishi-ku, Niigata, 950-2181 Japan \\ "lfengror@ku.ac.th
}

Received 23 January 2015

Accepted 2 April 2015

Published 20 May 2015

\begin{abstract}
A rapid predictive method based on near-infrared reflectance spectroscopy (NIRS) of paddy rice was developed to measure the pasting properties of rice. The paddy rice samples were scanned by a near-infrared reflectance spectrometer in the wavelength region of 1400-2400 nm and preprocessed by mathematical pretreatments prior to pasting properties analysis using a rapid visco-analyzer (RVA). The results indicated that the developed models of setback (SB), peak viscosity $(\mathrm{PV})$, breakdown $(\mathrm{BD})$ and consistency $(\mathrm{CS})$ provided good prediction results with relatively high correlation coefficients $(0.81-0.96)$. In addition, the validity of the calibration models was statistically tested. Standard error of prediction (SEP) and bias were small enough without any significance at $95 \%$ confidence interval. Nonetheless, this study proved that the use of NIRS for predicting pasting properties was feasible in paddy rice and could be applied in commercial trade and research.
\end{abstract}

Keywords: Pasting properties; near-infrared spectroscopy; paddy rice.

${ }{ }_{\text {Corresponding author. }}$

This is an Open Access article published by World Scientific Publishing Company. It is distributed under the terms of the Creative Commons Attribution 3.0 (CC-BY) License. Further distribution of this work is permitted, provided the original work is properly cited. 


\section{Introduction}

Typically, the most abundant component in rice is starch. It constitutes more than $80 \%$ of the total dry weight of polished rice. Starch is an important factor for determining the quality of rice products. ${ }^{1}$ In many Asian foods, such as noodles rice starch is the crucial ingredient. Starch pasting properties influence noodle quality, and are also directly responsible for starch industrial uses. ${ }^{2}$ Conventionally, the pasting properties of starch are measured by the rapid visco-analyzer (RVA). The analytical instrument was initially used to determine preharvest damage in cereals. The RVA had been used to provide information about pasting characteristics of starch of a particular sample under analysis (e.g., pasting peak viscosity (PV), final viscosity). ${ }^{3}$ One of the most important indices that are used to explain rice aging are pasting properties. During storage, changes of pasting properties are correlated to aging process individually. ${ }^{4}$ In terms of rice storage, changing trends of rice paste viscosity are different. ${ }^{5}$ For instance, the viscosity of rice paste increases considerably after short-to intermediate-term storage (months) of milled rice. ${ }^{6}$ However, it decreases during long-term storage (years). ${ }^{7}$ For RVA technique, in order to measure the pasting properties of rice, several steps of sample preparations and instruments are of major concern, since the method is time consuming and laborious.

A rapid technique, near-infrared reflectance spectroscopy (NIRS), was developed for measuring the quality characteristics that are routinely tested in cereals. ${ }^{8}$ In comparison to routine, conventional analysis methods, NIRS has many advantages. Firstly, sample preparation is simple and quick. Moreover complicated treatments or techniques are not needed for measurements. Second, it is a realtime determination of multiple characters with the development of calibration models. Thirdly, it is more flexible since the method can be applied to measure several kinds of samples. Fourthly, materials can be shipped and reused after measurements because the method is nondestructible. Lastly, the technique is economical; reagents and/or manpower are not needed. It is a clean and environmentfriendly analytical system since no wastes emerge. ${ }^{9}$

A few reports have been published on establishing the NIRS model for evaluating rice characteristics. Delwiche et $a l^{10}$ successfully used NIRS to determine milled whole-grain samples. Likewise,
Bao et al. ${ }^{11}$ also used visible reflectance and NIRS for determining milled rice flour samples and achieved satisfying prediction results. Moreover, Natsuga and Kawamura $^{12}$ studied pasting properties of brown and milled rice samples by visible reflectance and NIRS, and reported that the technique was an accurate tool for predicting pasting properties. Based on the aforementioned works, no previous research has reported the application of NIRS in paddy rice.

Therefore, this study was focused on Khoa Dawk Mali 105 paddy rice (cultivar KDML 105) as a material. The Khao Dawk Mali 105 rice is the most common rice cultivar in Thailand. It has a unique aroma and mild taste and is internationally recognized as "Jasmine Rice". In Thailand, KDML 105 rice is considered to be a vital crop for domestic consumption and primary export commodity for agricultural economic sector. ${ }^{13}$ Thailand is one of the world's largest KDML 105 rice producers and also the world's largest rice exporter in terms of rice amounts and cultivation area. Normally, the rice mills purchase paddy rice from farmers in order to process it into brown, parboiled and milled rice for export and domestic consumption according to market demands. Before trading and purchasing, qualities must be monitored for paddy pricing. Therefore, in this study, NIRS was used to measure pasting properties of paddy rice form, focusing on a nondestructive technique for measuring paddy rice quality.

\section{Materials and Methods}

\subsection{Rice samples}

264 rice samples (Khao Dawk Mali (KDML105) variety) were harvested from the Rice Seed Center in Chonburi Province which is the state agency of Thailand. Then, the samples were dried under the sun for a day. Moisture content of paddy rice samples ranged from $11 \%$ to $14 \%$ on wet basis. Subsequently, the samples were stored in a silo which had dimensions of $1.5 \times 1.5 \times 4.5 \mathrm{~m}$ (width $\times$ length $\times$ height), without controlling temperature. All samples $(1000 \mathrm{~kg})$ were kept throughout the storage period ( 6 months) after harvesting to study the change of pasting properties after the storage and extend ranges of pasting properties. Samples were taken once a month for the first 2 months of storage period. Afterward they were taken every 15 days until the end of the storage. 


\subsection{Spectroscopy analyses}

Entire paddy rice samples were scanned on a near infrared spectrophotometer (The InfraScan, BRUINS INSTRUMENTS, Puchheim, Germany). Approximately $70 \mathrm{~g}$ of a sample were loaded in a small ring quartz window-clad cylindrical cell (diameter $=$ $105 \mathrm{~mm}$, height $=15 \mathrm{~mm})$. The reflectance was measured in the NIR region within $1400-2400 \mathrm{~nm}$ and recorded during the rotation of the cylindrical cell at $0.5 \mathrm{~nm}$ intervals. Each sample was subsequently scanned, and average spectrum was collected (Fig. 1).

\subsection{Pasting properties}

After spectral acquisition, paddy rice samples were de-husked, milled, ground and sifted through a $0.8 \mathrm{~mm}$ sieve screen. Rice flour sample $(\sim 4.0 \mathrm{~g}$ of flour corrected using the moisture content of the sample, $\pm 0.01 \mathrm{~g})$ was mixed with distilled water $(\sim 25.0 \mathrm{~g}$ as the function of the amount of adjusted sample $\pm 0.01 \mathrm{~g}$ ) in an aluminum cylinder. The mixture was agitated by raising and lowering the plastic paddle through the aluminum cylinder before inserting the cylinder into the instrument. The pasting properties of slurries were determined with a rapid visco analyzer (RVA-4 Model, Newport Scientific Pty., Ltd., Warriewood NSW, Australia) in the RVU unit by performing the viscosity profile during 12.5-min heating cycle. The test profile had a starting temperature of $50^{\circ} \mathrm{C}$, which was maintained for $1 \mathrm{~min}$, later raised to $90^{\circ} \mathrm{C}$ within $4 \mathrm{~min}$, maintained for $10 \mathrm{~min}$, then cooled to $50^{\circ} \mathrm{C}$ in $1 \mathrm{~min}$, and maintained for $1 \mathrm{~min}$, with a stirring speed of $160 \mathrm{rpm}$ as in the test period. Properties were recorded and used for calculating other pasting

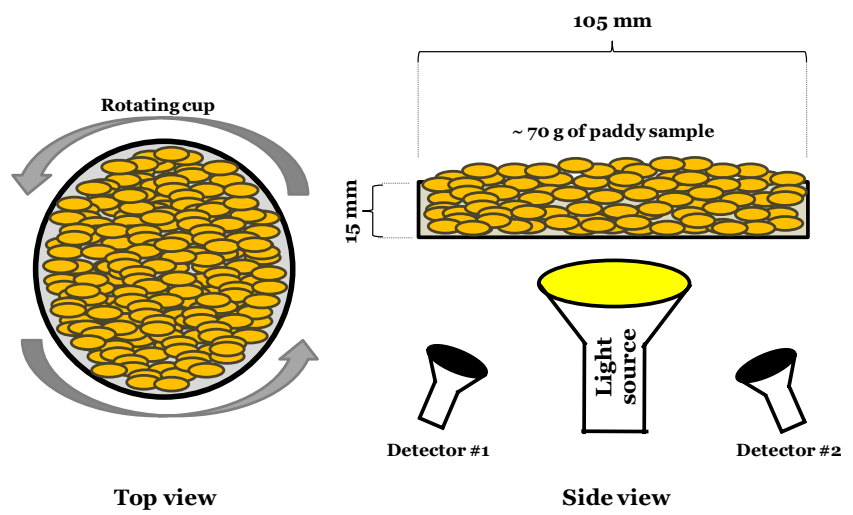

Fig. 1. Schematic diagram of reflectance NIRS measurement displaying in top view and side view. parameters such as $\mathrm{PV}$, hot paste viscosity (HV), cold paste viscosity $(\mathrm{CV})$, breakdown (BD), setback (SB) and consistency (CS). ${ }^{14}$

\subsection{Calibration and validation procedures}

Before calibration development, the reference value outliers were identified by Z-score. The outliers were limited to below $5 \%$ of samples unless the excess of outliers was removed. Spectral preprocessing was performed and calibration was developed based on partial least squares (PLS) regression with test set of a sample, using the software for multivariate analysis (Unscrambler version 9.8). A total of 160 samples were used as a calibration set and the rest (104 samples) was used as a validation set.

Statistic terms such as correlation of coefficients $(R)$; ratio of standard error of prediction (SEP) to standard deviation (RPD); SEP and standard deviation (SD) of difference between measured and predicted values; bias or average of difference between measured and predicted values of validation set were calculated. Moreover, validation test set was checked for reliability by following guidelines in ISO 12099:2010 ${ }^{15}$ as follows:

Firstly, $t$-test is checked by the significance of the bias. The calculation of the bias confidence limits (BCLS), the limit for accepting or rejecting model, is determined by $T_{b}$ which was calculated according to Eq. (1). If bias was smaller than $T_{b}$, then the bias obtained from the model had no significance or NIRS predicted values were not significantly different form measured values.

$$
T_{b}=\frac{t_{(1-\alpha / 2)} \mathrm{SEP}}{\sqrt{n}},
$$

where

$\alpha$ - is the probability of making a type-l error;

$t$ - is the appropriate Student $t$-value for a twotailed test with degrees of freedom associated with SEP and the selected probability of a type-I error;

$n$ - is the number of independent samples; SEP - is the standard error of prediction.

Secondly, the validity of the calibration model was checked by $F$-Test (ratio of variances) for SEP. The unexplained error confidence limits (UECLS), $T_{\mathrm{UE}}$ were calculated according to Eq. (2). If SEP was 
smaller than $T_{\mathrm{UE}}$ obtained from the model, SEP was in an acceptable range.

$$
T_{\mathrm{UE}}=\mathrm{SEC} \sqrt{F_{\alpha, \nu, M}},
$$

where

$$
\begin{aligned}
\text { SEC }- & \text { is the standard error of } \\
& \text { calibration; } \\
\nu=n_{v}-1- & \text { is the numerator; degrees of } \\
& \text { freedom associated with SEP } \\
& \text { of the test set in which } n \text { is } \\
& \text { the number of samples in the } \\
& \text { validation process; } \\
M=n_{c}-p-1- & \text { is the denominator; degrees of } \\
& \text { freedom associated with SEC } \\
n_{c}- & \text { is the number of calibration } \\
& \text { samples, } \\
p- & \text { is the number of terms or PLS } \\
& \text { factors in the model. }
\end{aligned}
$$

Lastly, the slope, $b$ of the sample regression: $y=$ $a+b \hat{y}$ with the reference value as the dependent variable and the predicted NIRS values as the independent variable was checked. From the least squares fitting, the slope is calculated as:

$$
b=\frac{S_{\hat{y} y}}{S_{\hat{y}}^{2}},
$$

where

$S_{\hat{y} y}$-is the covariance between measured and predicted values;

$S_{\hat{y}}^{2}$ - is the variance of the $n$ predicted values.

The intercept is calculated as:

$$
a=\bar{y}-b \overline{\hat{y}}
$$

where

$\overline{\hat{y}}$ - is the mean of the predicted values,

$\bar{y}$ - is the mean of the measured values,

$b-$ is the slope.

As for the bias, a $t$-test can be calculated to check the hypothesis that $b=1$

$$
t_{\mathrm{obs}}=|b-1| \sqrt{\frac{S_{\hat{y}}^{2}(n-1)}{S_{\mathrm{res}}^{2}}},
$$

where

$S_{\hat{y}}^{2}$ - is the variance of the $n$ predicted values

$S_{\text {res }}$ - is the residual SD as defined in Eq. (6)

$$
S_{\mathrm{res}}=\sqrt{\frac{\sum_{i=1}^{n}\left(y_{i}-a+b \hat{y}_{i}\right)^{2}}{n-2}}
$$

in which

$a$ - is the interception from Eq. (4),

$b$ - is the slope from Eq. (3),

$y_{i}$ - is the $i$ th measured value,

$\hat{y}_{i}$ - is the $i$ th predicted value obtained when applying the multivariate NIRS model.

The slope, $b$, is considered as significantly different from 1 when $t_{\mathrm{obs}} \geq t_{(1-\alpha / 2)}$ where

$t_{\mathrm{obs}}$ - is the observed $t$-value, calculated according to Eq. (5),

$t_{(1-\alpha / 2)}$ - is the $t$-value obtained for a probability of $\alpha=0.05(5 \%)$.

\section{Results and Discussion}

\subsection{Pasting properties change}

Pasting properties and sensitive index of the intrinsic properties of starchy rice materials are important. They ideally provide an excellent reflection of any changing phenomena that occur in the grain during storage. Changes of pasting properties are shown in Fig. 2. The PV, one of pasting properties, continually rose during the first 140 days and gradually declined until the end of studied period. For instance, in freshly harvested rice, the increase in PV during early period of storage (few months) was influenced by the presence of amylase activity. However, later the PV gradually declined due to the lack of amylase activity. ${ }^{16}$ In case of the SB, it notably increased during 185 days of storage and slightly changed afterward. A similar trend was observed for the cold paste viscosity, after storing for 185 days, the value declined and was close to the

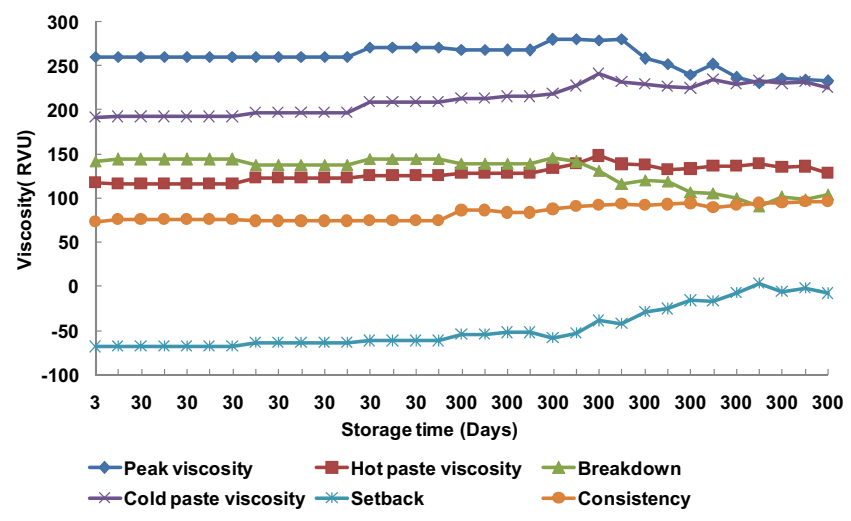

Fig. 2. The change of pasting properties in KDML105 cultivar after storage. 
Table 1. Pasting properties of rice samples throughout six months storage period.

\begin{tabular}{lcccccc}
\hline Property & Unit & $n$ & Mean & Min & Max & SD \\
\hline $\begin{array}{l}\text { Peak viscosity } \\
\quad \text { PV) }\end{array}$ & RVU & 264 & 264.83 & 209.83 & 310.50 & 17.09 \\
$\begin{array}{l}\text { Hot paste } \\
\quad \text { viscosity (HV) }\end{array}$ & RVU & 264 & 134.94 & 107.67 & 164.83 & 8.92 \\
$\begin{array}{l}\text { Cold paste } \\
\quad \text { viscosity (CV) }\end{array}$ & RVU & 264 & 129.89 & 87.92 & 173.75 & 15.76 \\
$\begin{array}{l}\text { Breakdown (BD) } \\
\text { Setback (SB) }\end{array}$ & RVU & 264 & 224.79 & 175.33 & 253.58 & 13.78 \\
Consistency (CS) & RVU & 264 & -39.90 & -81.08 & -4.75 & 18.84 \\
\hline
\end{tabular}

Note: RVU; rapid visco-analyzer units.

value observed in fresh rice. There was a significant change in BD at all storage times. This change can be attributed to the uniqueness of the starch granules. The decrease in $\mathrm{BD}$ value indicated that the ability of the starch granules to BD after cooking significantly reduced by aging of the granules. The increase in PV showed that the starch granules of storage rice were more resistant to swelling than those of fresh rice.

Table 1 shows the mean, minimum, maximum and SD values of the pasting properties of rice samples. Typically, in order to develop an effective model for quality prediction, the variations such as cultivars and harvest time should be included in datasets. In this experiment, we used only one cultivar, but the data variations were dependent on storage time.

\section{2. $\quad$ Predicting pasting properties}

\subsubsection{Pretreatment procedure}

The original NIR reflectance spectra of paddy rice samples in long wavelength region (1400-2400 nm) are shown in Fig. 3. According to the chemical assignments, $\mathrm{H}_{2} \mathrm{O}$ molecule could absorb at $1450 \mathrm{~nm}$ (O-H stretching 1st overtone) and $1940 \mathrm{~nm}(\mathrm{O}-\mathrm{H}$ stretching $+\mathrm{O}-\mathrm{H}$ deformation). As for starch molecule, the chemical bond vibrations were also observed at 1450 and $2100 \mathrm{~nm}(2 \times \mathrm{O}-\mathrm{H}$ deformation + $2 \times \mathrm{C}-\mathrm{O}$ stretching) previously described by Osborne et al. In our study, spectral characteristic notably revealed baseline shift phenomenon. In addition, after the radiation encountered discrete particle within the sample, destructive interference became incomplete and the radiation propagated in all directions. It is known as scattering. This was due to the random

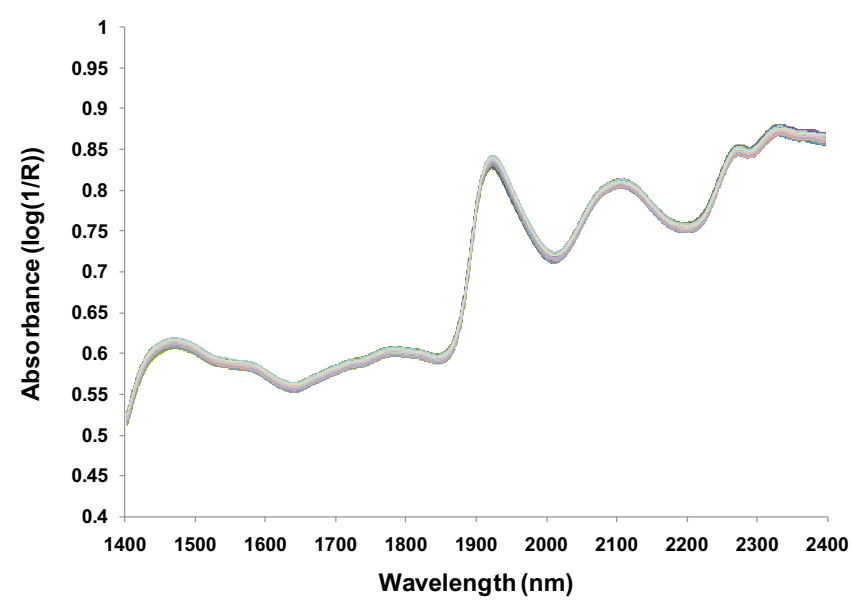

Fig. 3. NIR whole spectra of KDML 105 variety paddy rice samples in wavelength range $1400-2400 \mathrm{~nm}$.

diffusion in reflectance measurements since the radiation is reflected, diffused and scattered at further sample interface. In case of rice samples, macrostructure of rice intensively affected the random optical path length because the light was transmitted to the rice and reflected randomly among rice kernels which led to scattering. Therefore, various NIR spectra pretreatments were employed in this study, namely Savitzky-Goley first derivative and standard normal variant (SNV).

\subsubsection{PLS results}

The results of PLS models for predicting pasting properties from paddy rice spectra are shown in Table 2. Performance was evaluated using the correlation coefficient $(R)$ between measured values and predicted NIRS values; the SEP; RPD and bias. The best PLS of a model developed for predicting SB was obtained from the Savitzky-Golay smoothing (gap $1.25 \mathrm{~nm}$ ) and SNV in $1400-2250 \mathrm{~nm}$ range. A value of $R$ of pasting properties of rice (SB) gave good results, providing high $R(0.96)$, small SEP (6.54) and low bias (0.58). Among them, higher RPDs $(>2)$ were mostly obtained for PV (2.34), CV (2.02), BD (2.14), SB (3.52) and CS (2.30). Only HV (1.82) had a relatively low RPD. The RPD was explained by Fearn. ${ }^{17}$ Calibration of calculated RPD between 2 and 10 proved to be a good model resulting in dependable predicting result. The results in Table 2 also indicated those reasonable models $(R>0.8)$ that were obtained from the use of wavelength ranges regions between 1400 and $2250 \mathrm{~nm}$. This region typically has high 


\section{P. Siriphollakul et al.}

Table 2. PLS models result for predicting pasting properties of paddy rice using difference mathematical pretreatments and wavelength range selections.

\begin{tabular}{lcccccc}
\hline Property & Pretreatment & Range & $R$ & SEP (RVU) & RPD & Bias (RVU) \\
\hline Peak viscosity (PV) & First derivatives & $1400-2250$ & 0.90 & 8.50 & 2.34 & -1 \\
Hot paste viscosity (HV) & SNV & $1400-2000$ & 0.83 & 4.00 & 1.82 & -0.60 \\
Cold paste viscosity (CV) & First derivatives +SNV & $1400-2000$ & 0.85 & 4.09 & 2.02 & 1.51 \\
Breakdown (BD) & SNV & $1400-2250$ & 0.88 & 8.97 & 2.14 & 1.11 \\
Setback (SB) & SNV & $1400-2250$ & 0.96 & 6.54 & 3.52 & 0.58 \\
Consistency (CS) & SNV & $1400-2250$ & 0.81 & 2.09 & 2.30 & 0.09 \\
\hline
\end{tabular}

Note: $R$; correlation of coefficient, SEP; standard error of prediction, RPD; ratio of standard error of prediction to standard deviation and bias; average error of prediction.

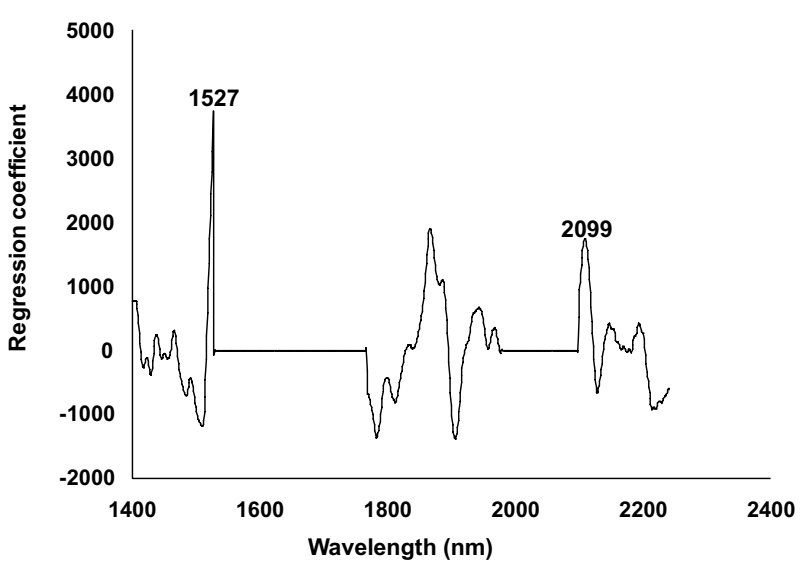

Fig. 4. The PLS model coefficients of model developed for predicting setback of paddy rice in wavelength range of 1400$2400 \mathrm{~nm}$.

molar absorptivity. The regression coefficients of the developed model for predicting SB using six factors are presented in Fig. 4. The regression analysis also revealed the highest relationship at
1527 and $2099 \mathrm{~nm}$ wavelength. Osborne ${ }^{8}$ reported that wavelengths of 1528 and $2100 \mathrm{~nm}$ are typically responsible for starch, the major component of rice. The pasting quality of rice is basically dictated by the quality of the starch. Pasting properties effectively explained the behavior of rice flour and starch during processing (heating and/or cooling). For other studied properties, absorbance of starch was shown to be highly influenced in regression coefficient plots of all models. Figures $5(\mathrm{a})-5(\mathrm{f})$ showed scatter plots of the measured and predicted values of the pasting properties for validation set. Dots scattered close to target line (diagonal line) indicate that NIRS could predict all pasting properties accurately or predicted values were highly correlated to measured values.

Furthermore, the aforementioned incidences were confirmed by ISO indices. R, SEP and bias were used to consider model accuracy and performance according to ISO 12099. Firstly, $T_{b}$, bias confidence limits for a probability of 0.05 , one of the

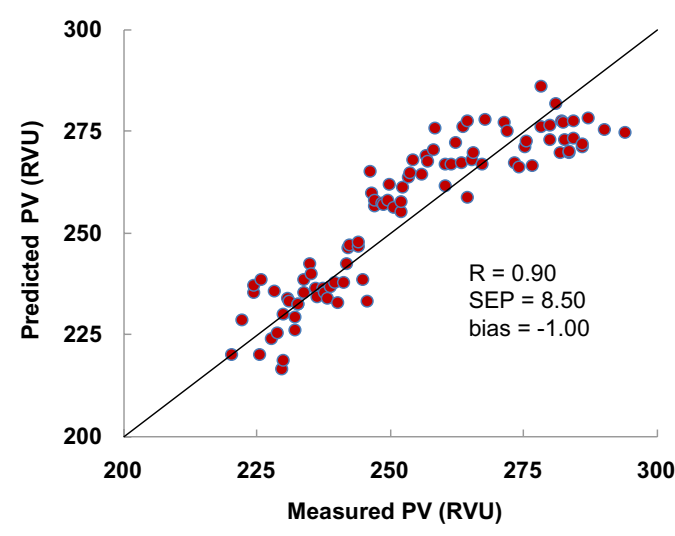

(a)

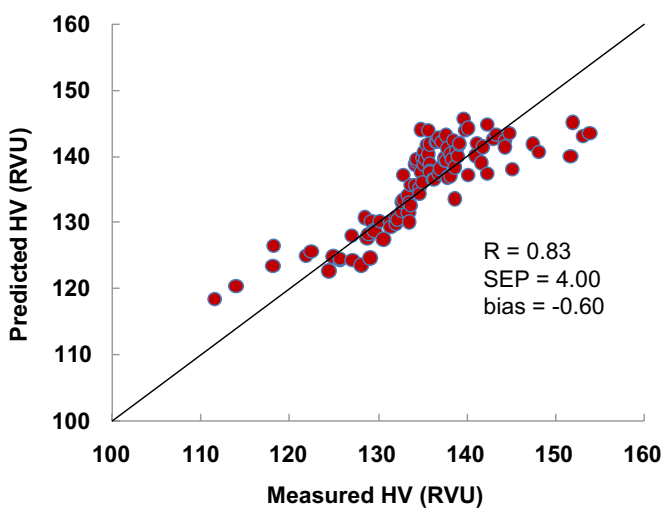

(b)

Fig. 5. Comparison of measured and predicted values of pasting properties by PLS model for validation set (a) peak viscosity $(\mathrm{PV}),(\mathrm{b})$ hot paste viscosity $(\mathrm{HV}),(\mathrm{c})$ cold paste viscosity $(\mathrm{CV}),(\mathrm{d})$ breakdown (BD), (e) setback (SB) and (f) consistency (CS) 


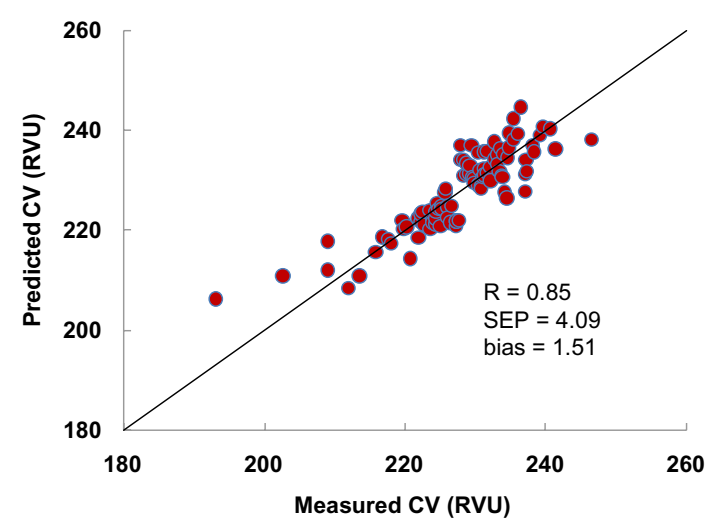

(c)

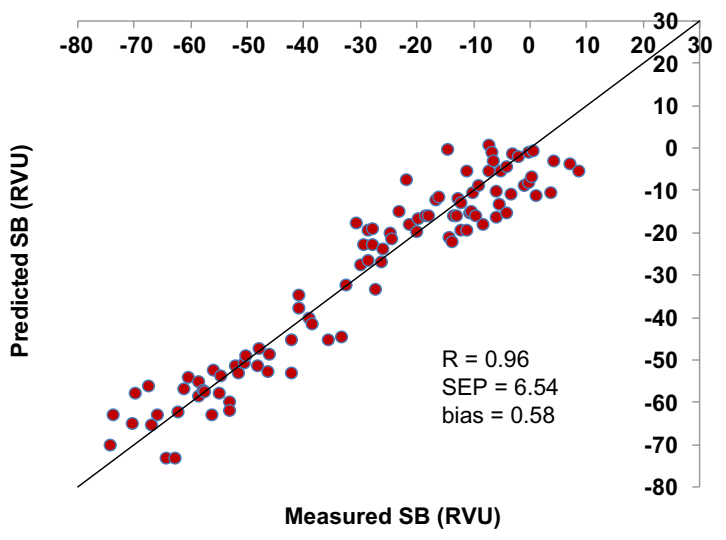

(e)

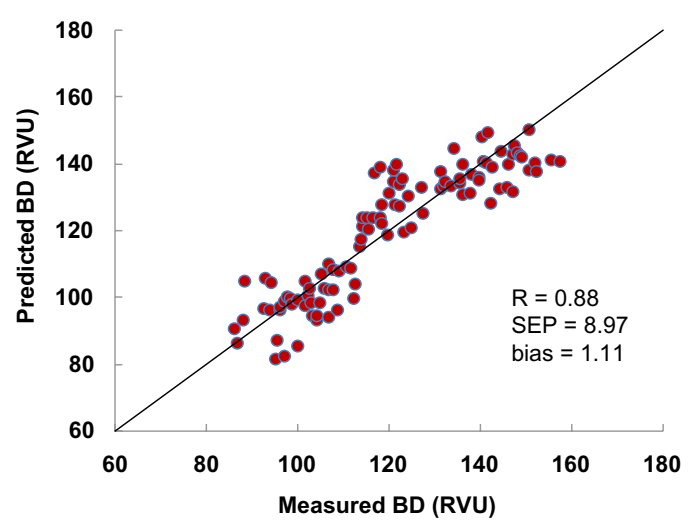

(d)

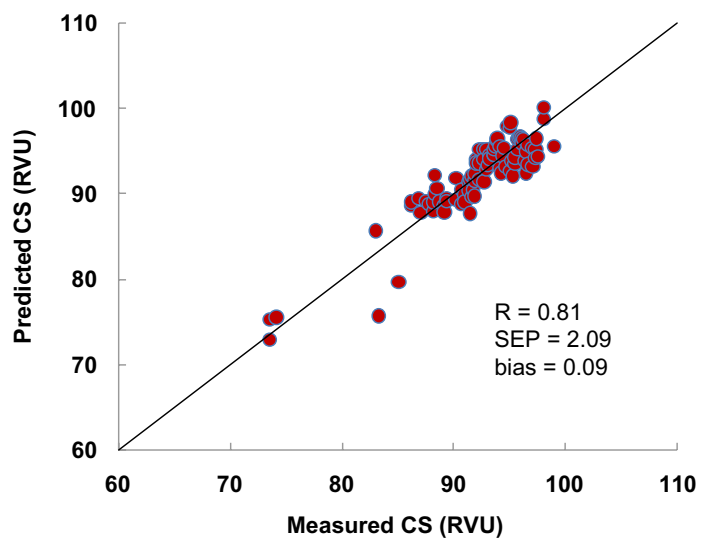

(f)

Fig. 5. (Continued)

Table 3. The statistic test of the significance of bias for model performance.

\begin{tabular}{lrcc}
\hline Property & \multicolumn{1}{c}{$T_{b}^{\mathrm{a}}$} & Bias (RVU) & Result \\
\hline Peak viscosity (PV) & -1.32 & -1 & $\checkmark$ \\
Hot paste viscosity (HV) & -1.53 & -0.60 & $\checkmark$ \\
Cold paste viscosity (CV) & 2.94 & 1.51 & $\checkmark$ \\
Breakdown (BD) & 1.17 & 1.11 & $\checkmark$ \\
Setback (SB) & 0.88 & 0.58 & $\checkmark$ \\
Consistency (CS) & 0.41 & 0.09 & $\checkmark$ \\
\hline
\end{tabular}

aThe significance of bias is checked by $t$-test. If bias is smaller than $T_{b}$, then the bias obtained from the model has no significance at $95 \%$ confident interval.

important statistic terms, were calculated and compared. Table 3 showed that all bias values were smaller than $T_{b}$ values indicating that the bias of developed models was considered as not different from zero. Secondly, $T_{\mathrm{UE}}$ the unexplained error confidence limits were determined. All models in
Table 4. The statistic test of SD of the residuals for model performance.

\begin{tabular}{lrcc}
\hline Property & \multicolumn{1}{c}{$T_{\mathrm{UE}}^{\mathrm{a}}$} & SEP (RVU) & Result \\
\hline Peak viscosity (PV) & 15.43 & 8.50 & $\checkmark$ \\
Hot paste viscosity (HV) & 5.82 & 4.00 & $\checkmark$ \\
Cold paste viscosity (CV) & 7.52 & 4.09 & $\checkmark$ \\
Breakdown (BD) & 9.80 & 8.44 & $\checkmark$ \\
Setback (SB) & 11.04 & 6.54 & $\checkmark$ \\
Consistency (CS) & 4.19 & 2.09 & $\checkmark$ \\
\hline
\end{tabular}

a The unexplained error confidence limit, $T_{\mathrm{UE}}$ is calculated from an $F$-test. If SEP is smaller than $T_{\mathrm{UE}}$, then SEP obtained from the model is in an acceptable range at $95 \%$ confident interval.

Table 4 had $T_{\mathrm{UE}}$ larger than SEP obtained from the model indicating that SEP was small enough and in an acceptable range. Lastly, the slope $(b)$ was considered (Table 5). The condition of $t_{\mathrm{obs}}>$ $t(1-\alpha / 2)$ when the $b$ was different from 1 , while 
Table 5. The statistic test of slope of models development.

\begin{tabular}{lcccc}
\hline Property & Slope $^{\mathrm{a}}$ & $t_{\text {obs }}^{\mathrm{b}}$ & $t(1-\alpha / 2)^{\mathrm{c}}$ & Result \\
\hline Peak viscosity (PV) & 0.848 & 0.063 & 1.99 & $\checkmark$ \\
Hot paste viscosity (HV) & 0.761 & 0.077 & 1.99 & $\checkmark$ \\
Cold paste viscosity (CV) & 0.852 & 0.029 & 1.99 & $\checkmark$ \\
Breakdown (BD) & 0.897 & 0.909 & 1.99 & $\checkmark$ \\
Setback (SB) & 0.915 & 0.271 & 1.99 & $\checkmark$ \\
Consistency (CS) & 0.928 & 0.019 & 1.99 & $\checkmark$ \\
\hline
\end{tabular}

a Slope obtained from the regression of the measured values and the predicted values.

bThe observed $t$-value.

${ }^{c}$ The $t$-value obtained for a probability of $\alpha=0.05$ (5\%) when $t_{\mathrm{obs}}<t(1-\alpha / 2)$, slope is not significance from 1 .

$t_{\text {obs }}<t(1-\alpha / 2)$ showed a successful prediction or $b$ was looked upon as 1 . Our results showed that slopes of all models were not significantly different from 1. Therefore, developed models for predicting pasting properties of paddy rice were accomplished according to ISO 12099.

\section{Conclusion}

Nondestructive measurement of pasting properties of paddy rice was studied using NIRS. The technique performed well for predicting the pasting properties with satisfactory performance as the validity of the calibration models was statistically tested. This study proved that the use of NIRS is suitable for predicting paddy rice pasting properties.

\section{Acknowledgments}

The authors would like to thank King Mongkut's University of Technology Thonburi for materials funds, Rajamungla University of Technology TawanOk for the Near Infrared Spectrometer and the NIR Technology laboratory of Kasetsart University for Unscrambler program version 9.8.

\section{References}

1. J. Hasjim, E. Li, S. Dhital, "Milling of rice grains: Effects of starch/flour structures on gelatinization and pasting properties," Carbohydr. Polym. 92, 682-690 (2013).

2. L. S. Collado, R. C. Mabesa, H. Corke, "Genetic variation in the physical properties of sweet potato starch", J. Agric. Food Chem. 47, 4195-4201 (1999).

3. D. Cozzolino, K. Allder, S. Roumeliotis, J. Eglinton, "Feasibility study on the use of multivariate data methods and derivatives to enhance information from barley flour and malt samples analyzed using the rapid visco analyser," J. Cereal Sci. 56, 610-614 (2012).

4. A. A. Perdon, B. P. Marks, T. J. Siebenmorgen, N. B. Reid, "Effects of rough rice storage conditions on the amylograph and cooking properties of medium-grain rice cv. Bengal," Cereal Chem. 74, 864867 (1997).

5. W. M. Lin, S. M. Chang, C. Y. Li, "Studies on the changes of physicochemical properties of rice during storage," Acad. Sin. 26, 13-23 (1979).

6. N. Shibuya, T. Iwasaki, H. Yanase, S. Chikuba, "Studies on determination of rice during storage I: Changes of brown rice and milled rice during storage," J. Japanese Soc. Food Sci. Technol. 21, 597-603 (1974).

7. C. M. Sowbhagya, K. R. Bhattacharyat, "Changes in pasting behavior of rice during ageing," J. Cereal Sci. 34, 115-124 (2001).

8. B. G. Osborne, "Applications of near infrared spectroscopy in quality screening of early-generation materials in cereal breeding programmers," J. Near Infrared Spectrosc. 14, 93-100 (2006).

9. L. Guoquan, H. Huahong, Z. Dapeng, "Prediction of sweet potato starch physiochemical quality and pasting properties using near-infrared reflectance spectroscopy," Food Chem. 94, 632-639 (2006).

10. S. R. Delwiche, K. S. McKenzie, B. D. Webb, "Quality characteristics in rice by near-infrared reflectance analysis of whole-grain milled samples," Cereal Chem. 73, 257-263 (1996).

11. J. S. Bao, Y. Z. Cai, H. Corke, "Prediction of rice starch quality parameters by near-Infrared reflectance spectroscopy," Food Chem. Toxicol. 66, 936-939 (2001).

12. M. Natsuga, S. Kawamura, "Visible and near infrared reflectance spectroscopy for determining physicochemical properties of rice," Trans. ASABE 49(4), 1069-1076 (2006).

13. S. Chitrakorn, "Rice and the Thai way of life," Science and Technology with Thai Rice, S. Lorlowhakarn, Ed., pp. 13-22, Public Information Department Thailand's National Science and Technology Development Agency, Bangkok (2003).

14. AACC International, Approved Methods of the American Association of Cereal Chemists, 10th Edition, Methods 61-02. The Association: St. Paul, MN (2000).

15. International Standards Organization (ISO) 12099, Animal Feeding Stuffs, Cereals and Milled Cereal Products-Guidelines for the Application of Near Infrared Spectrometry, pp. 6-11 (2010).

16. K. R. Bhattacharya, Rice Quality, A Guide to Rice Quality and Analysis, pp. 578, Woodhead Publishing Limited, UK (2011).

17. T. Fearn, Assessing calibrations: SEP, RPD, RER and $\mathrm{R}^{2}$, NIR News 13, 12-14 (2002). 